\title{
LOW LEVEL LASER THERAPY FOR PATIENTS WITH CERVICAL DISK HERNIA
}

\author{
Hiroshi Takahashi ${ }^{1}$, Ikuko Okuni ${ }^{2}$, Nobuyuki Ushigome ${ }^{2}$, Takashi Harada ${ }^{2}$, \\ Hiroshi Tsuruoka ${ }^{2}$,Toshio Ohshiro ${ }^{3}$, Masayuki Sekiguchi ${ }^{1}$,Yoshiro Musya ${ }^{4}$ \\ 1: Department of Orthopaedic Surgery Toho University School of Medicine \\ 2: Department of Rehabilitation Medicine Toho University School of Medicine \\ 3: Japan Medical Laser Laboratory, Shinjuku-ku Tokyo Japan \\ 4: Department of Orthopaedic Surgery Ohashi Hospital Toho University School of Medicine
}

\begin{abstract}
Background and Aims: In previous studies we have reported the benefits of low level laser therapy (LLLT) for chronic shoulder joint pain, elbow, hand and finger pain, and low back pain. The present study is a report on the effects of LLLT for chronic neck pain.

Materials and Methods: Over a 3 year period, 26 rehabilitation department outpatients with chronic neck pain, diagnosed as being caused by cervical disk hernia, underwent treatment applied to the painful area with a $1000 \mathrm{~mW}$ semi-conductor laser device delivering at $830 \mathrm{~nm}$ in continuous wave, $20.1 \mathrm{~J} / \mathrm{cm}^{2} /$ point, and three shots were given per session (1 treatment) with twice a week for 4 weeks.

\section{Results:}

1. A visual analogue scale (VAS) was used to determine the effects of LLLT for chronic pain and after the end of the treatment regimen a significant improvement was observed $(\mathrm{p}<0.001)$.

2. After treatment, no significant differences in cervical spine range of motion were observed.

3. Discussions with the patients revealed that in order to receive continued benefits from treatment, it was important for them to be taught how to avoid postures that would cause them neck pain in everyday life.

Conclusion: The present study demonstrates that LLLT was an effective form of treatment for neck and back pain caused by cervical disk hernia, reinforced by postural training.
\end{abstract}

Key words: Low Level Laser Therapy, Cervical Disk Hernia, Chronic Pain, Postural training during Activities of Daily Living

\section{Introduction}

Cervical spondylosis is a disease characterised by intervertebral disk degeneration of the cervical spine and symptoms can be classified into three groups: 1 . local signs, 2. cervical disk hernia, and 3. cervical myelopathy. 1

Based on the many reports in the literature on the efficacy of LLLT for various musculoskeletal pain

Addressee for Correspondence:

Takashi Harada

Address: Department of Rehabilitation Medicine, Toho

University School of Medicine 6-11-1 Ohmori-nishi,

Ohtaku,Tokyo 143-8541 Japan

TEL:+81-3-3762-4151 FAX:+81-3-3768-6117

E-mail:haradat-3522@yahoo.co.jp types and on our own previous experiences with LLLT for pain in other areas, we surmised that low level laser therapy (LLLT) could be a suitable treatment for pain associated with cervical disk hernia. We also believed that spinal cord signs associated with cervical myelopathy, including spasticity, may be reduced with LLLT. However, there were only a few cases of cervical myelopathy observed in this study, therefore, results related to cervical myelopathy were excluded.

Pain caused by cervical disk hernia tends to be localised in the neck and shoulder joints but referred pain in the back region is also observed when the cervical disk hernia is extended. The purpose of this study

Manuscript received: July 30th, 2012

Accepted for publication: September 7th, 2012 
was to investigate the effectiveness of LLLT as a method of treatment for cervical disk hernia.

\section{Subjects and Methods}

\section{Subjects}

15 males and 11 females between the age of 36 and 76 (average age $55.5 \mathrm{yr}$ ) took part in this study. All subjects were outpatients who visited the rehabilitation department of our hospital between April 2005 and March 2008, with a confirmed diagnosis of cervical disk hernia (chronic type) (Table 1). Diagnosis of the disease was based on the X-ray findings, MRI and physical symptoms.

Table 1: Outline of Cases

\begin{tabular}{|c|c|c|c|c|c|}
\hline \multirow[t]{2}{*}{ Case } & \multirow[t]{2}{*}{ Age } & \multirow[t]{2}{*}{ Sex } & \multirow{2}{*}{$\begin{array}{l}\text { Treatment targets } \\
\quad \text { (See fig. } 2)\end{array}$} & \multicolumn{2}{|c|}{ VAS scores } \\
\hline & & & & before & after \\
\hline $1:$ & 57 & $\mathrm{M}$ & A B C & 70 & 45 \\
\hline $2:$ & 76 & $\mathrm{M}$ & A B & 60 & 40 \\
\hline 3: & 67 & $\mathrm{~F}$ & A B E & 55 & 40 \\
\hline 4: & 36 & $\mathrm{M}$ & A B D & 85 & 30 \\
\hline 5: & 65 & $\mathrm{~F}$ & A B & 40 & 35 \\
\hline $6:$ & 63 & M & A B D & 50 & 30 \\
\hline $7:$ & 54 & $\mathrm{~F}$ & A B C & 60 & 40 \\
\hline 8: & 59 & M & A B E & 90 & 50 \\
\hline 9: & 58 & F & A B & 60 & 30 \\
\hline 10: & 56 & M & A B C & 70 & 45 \\
\hline 11: & 46 & $\mathrm{~F}$ & A B C & 50 & 30 \\
\hline 12: & 51 & M & A B & 75 & 30 \\
\hline 13: & 55 & M & A B D & 70 & 45 \\
\hline 14: & 54 & $\mathrm{~F}$ & A B E & 90 & 50 \\
\hline 15: & 64 & M & A B E & 70 & 45 \\
\hline 16: & 55 & $\mathrm{~F}$ & A B E & 70 & 35 \\
\hline 17: & 55 & M & A B E & 60 & 40 \\
\hline 18: & 51 & M & A B E & 70 & 15 \\
\hline 19: & 37 & $\mathrm{~F}$ & A B D & 45 & 20 \\
\hline 20: & 79 & M & A B E & 60 & 40 \\
\hline 21: & 38 & F & A B C & 90 & 50 \\
\hline 22: & 44 & $\mathrm{~F}$ & A B E & 70 & 40 \\
\hline 23: & 42 & $\mathrm{M}$ & A B D & 70 & 35 \\
\hline 24: & 59 & $\mathrm{M}$ & A B & 50 & 40 \\
\hline $25:$ & 70 & $\mathrm{~F}$ & A B D & 70 & 45 \\
\hline 26: & 52 & $\mathrm{M}$ & A B & 50 & 45 \\
\hline
\end{tabular}

\section{LaserTherapy Device and Irradiation Method}

LLLT was administered with a semi-conductor therapeutic laser system (Figure 1) the MDL-2001 (Matsushita Electric Corporation, Tokyo, Japan) delivering $1000 \mathrm{~mW}$ at $830 \mathrm{~nm}$ in continuous wave with an irradiated area of $1.5 \mathrm{~cm}^{2}$ (full specifications in Table 2). The target areas on the relevant muscles where LLLT was applied are shown in Figure 2, and their

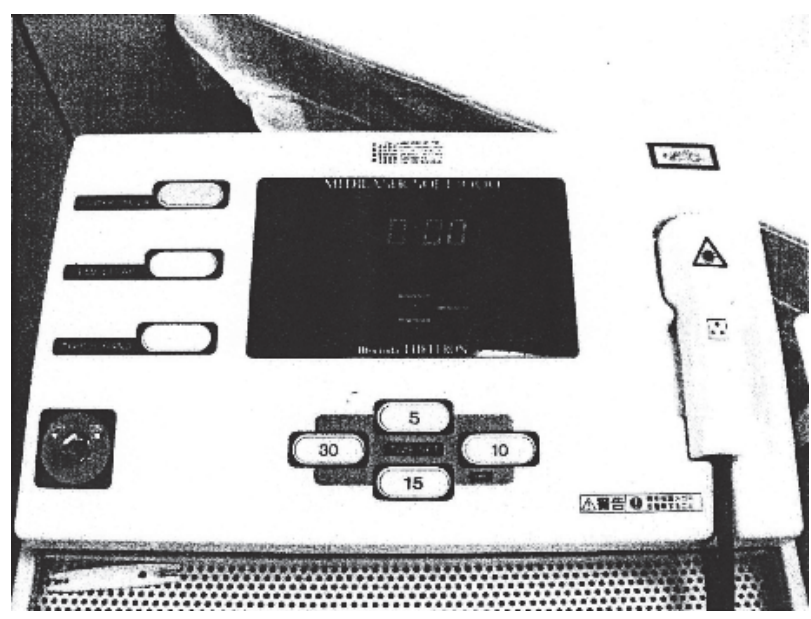

Fig. 1: The low level laser therapy device used in the present study

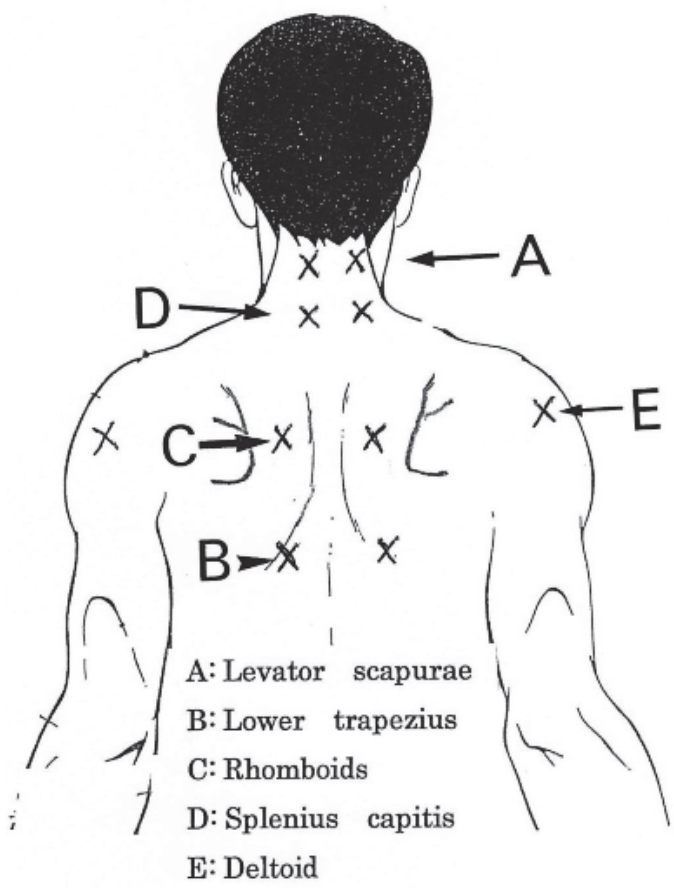

Fig. 2: Target points A - E for LLLT irradiation (shown by ' $x$ '). Note that all points are bilateral. 
Table 2: Specification of the low level laser therapy device used in the present study

\begin{tabular}{ll}
\hline Laser type and active medium & Semiconductor Laser: gallium aluminum arsenide (GaAlAs) diode \\
Model \& Manufacturer & MDL-2001 \\
& Matsushita Electric Corporation, Tokyo, Japan \\
Wavelength & $830 \mathrm{~nm} \pm 15 \mathrm{~nm}$ \\
Output & $1000 \mathrm{~mW} \pm 20 \%$ \\
Mode & Continuous wave mode \\
& Contact method with positive pressure \\
Beam diameter and treatment area & Diameter, $14 \mathrm{~mm}$ \\
& Irradiated area, approx. $1.5 \mathrm{~cm}^{2}$ \\
Irradiance & Approx $650 \mathrm{~mW} / \mathrm{cm}^{2}$ \\
Irradiation time & $30 \mathrm{~s}$ \\
Energy density & $19.5 \mathrm{~J} / \mathrm{cm}^{2}[0.650 \mathrm{x} 30=19.5]$ \\
Power supply & $100 \mathrm{VAC}, 50-60 \mathrm{HzM}$ \\
\hline
\end{tabular}

respective segmental spinal innervation is shown in Table 3. The laser was applied to each target area in the contact mode applying gentle pressure for $30 \mathrm{sec}-$ onds per point, giving an incident energy density of 19. $5 \mathrm{~J} / \mathrm{cm}^{2}$, and typically $4-6$ areas of irradiation were treated per patient. The total time taken for 1 treatment was 2-3 minutes. We irradiated the same target points three times each. Treatment sessions were a week for 4 weeks.

\section{Evaluation of Pain and Cervical Spine Range of Motion (ROM)}

Pain was assessed after treatment using a Visual Analogue Scale (VAS). Also, after treatment, cervical spine flexion and extension mobility levels were measured to assess any changes in ROM values.

\section{Lifestyle Guidance}

Patients were advised to follow their normal living

Table 3: Muscles listed according to their spinal segment innervation

\begin{tabular}{lcccccc}
\hline Involved muscles & \multicolumn{7}{c}{ Spinal Segment (Cervical) } \\
& $\mathbf{3}$ & $\mathbf{4}$ & 5 & $\mathbf{6}$ & $\mathbf{7}$ & $\mathbf{8}$ \\
\hline A: Levator scapulae & $\circ$ & $\circ$ & $\circ$ & & & \\
B: Lower trapezius & $\circ$ & $\circ$ & & & & \\
C: Rhomboid & & $\circ$ & $\circ$ & & & \\
D: Splenius capitis & & $\circ$ & $\circ$ & $\circ$ & $\circ$ & $\circ$ \\
E: Deltoid & & & $\circ$ & $\circ$ & & \\
\hline
\end{tabular}

style. We give them written advice sheets on maintaining a good posture, to avoid slouching in chairs or when sitting at a table or desk, to perform gentle head and neck rotation and extension exercises daily, and so on.

\section{Statistical Analysis}

VAS was measured before treatment started (baseline) and after the final 8 full LLLT sessions, and these two sets of scores were averaged and compared. All values were expressed as the means \pm standard deviation. Comparisons of values were performed with the Wilcoxon signed rank test (nonparametric score), using SPSS Statistics Ver. 17.0J, with a value of less than $5 \%$ considered significant.

The study was conducted under the principles of the Declaration of Helsinki (2004). The trial was conducted with the approval of the Ethics Committee of the Toho University School of Medicine Institutional Review Board (IRB). The purpose and potential outcomes of the trial were explained to all participants, whereafter they gave written informed consent to participate in the study.

\section{Results}

\section{Pain Evaluation}

The VAS scores at baseline and at the final assessment are shown in Table 1, and Figure 3. The average VAS scores before treatment were $65.4 \pm 13.8($ mean + SD) and after treatment were $38.1 \pm 8.8$. The average drop 


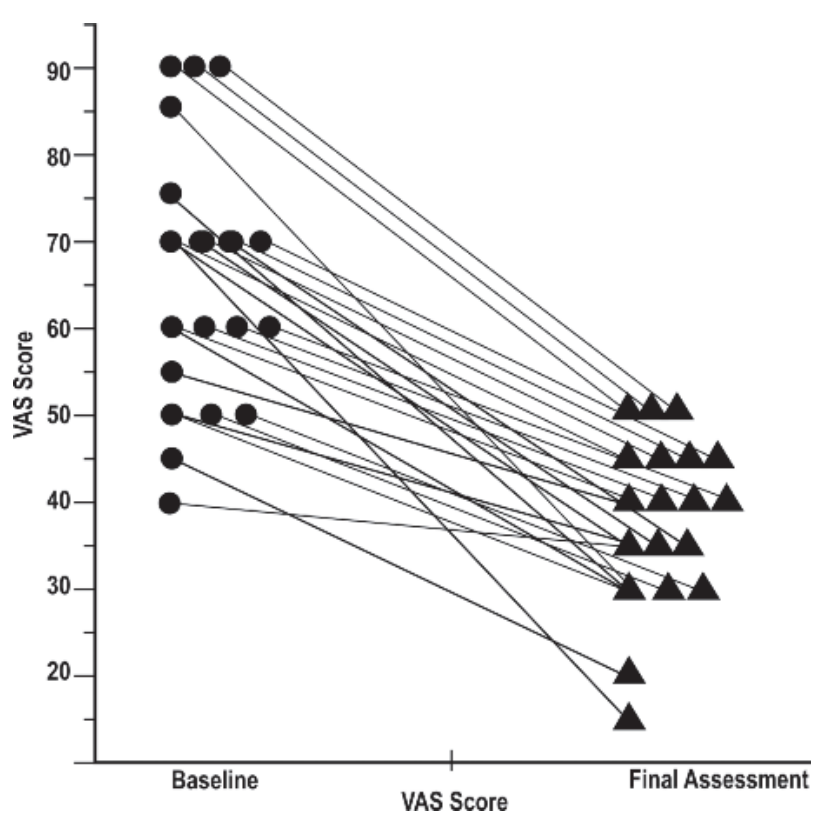

Fig. 3: Scatter plot showing uniform reduction in pain VAS score for all patients. Min, 5 points; max, 55 points; mean $26.92 \pm 13.2$ points $(\mathrm{p}<0.001)$

was $26.92 \pm 13.2$ points. A significant improvement was observed ( $\mathrm{p}<0.001$ for both).

\section{Cervical Spine Joint Mobility}

Cervical spine extension and flexion were measured but no significant differences were observed after LLLT treatment (data not shown).

\section{Patient Lifestyle Guidance}

Patients were advised to avoid postures that induce pain and we endeavored to guide patients on how to avoid incorrect positions in their Activities of Daily Living (ADL) as already mentioned in the Materials and Methods section. In discussions with the patients, it was reported in all cases that the lifestyle guidance we provided was understood and the advice that was given was followed. However, in the score-sheet filled out by patients after the treatment, there were many cases where patients had not precisely followed the lifestyle guidelines, therefore statistical analysis was not performed on this parameter.

\section{Discussion}

There has been much basic research ${ }^{2-6}$ and many clinical studies ${ }^{7-11}$ carried out on LLLT. Some reports dis- cuss types of LLLT devices ${ }^{12}$, wavelength-specific benefits 12,13 and evaluation of treatment methods. 13,14 Recently, basic research on LLLT is gaining validity. ${ }^{15-}$ 17

The frequency at which cervical spondylosis is observed in rehabilitation outpatients is high. The prognosis is good for patients with neck pain and cervical disk hernia. However, the prognosis for cases involving spinal cord signs (cervical myelopathy) is believed to be generally poor, and such patients were not evaluated in the presents study because of low numbers. On the other hand, the present study confirms the beneficial effects of LLLT treatment for neck pain originating from cervical disk hernia.

Countermeasures to reduce pain are important as pain experienced by patients reduces their efficiency at work and/or is a hindrance in their ADL. The mainstream treatment protocols prescribed for outpatients with cervical disk hernia in orthopaedic departments are relaxation treatments, along with medication and cervical traction. ${ }^{17,18}$

Due to changes associated with age, cervical disk hernia is more commonly induced in middle-aged to elderly people. The burden of cervical disk hernia in the elderly, especially those on medication for illnesses other than cervical disk hernia, is particularly high. Also, during cervical spine traction, it is difficult to control the force of traction and there are also cases where it is difficult to control neck posture. Furthermore, there are even cases where traction worsens neck pain. On the other hand, LLLT treatment is simple and easy to administer with no side effects. The satisfaction level of patients is also very high.

Pain as a result of work-related posture, e.g., sitting at a desk or working on a computer, and pain caused by unnatural positions in ADL, exacerbates pain caused by cervical disk hernia. Therefore, guidance was given to patients on how to avoid such positions in daily life.

Regarding the evaluation of pain, in the present study a significant effect was observed in the VAS score measured after irradiation $(\mathrm{p}<0.001)$. However, there was no significant change in cervical spine range of motion after LLLT treatment.

There are various possible explanations for the positive effects of LLLT treatment. The authors believe that a reduction in muscle tone, improved blood flow, a rise in the pain threshold and regulation by the autonomic nervous system are the main contributing factors.

In cases where LLLT does not provide sufficient results, we believe the second line of treatment should 
be with orthoses. Furthermore, in serious cases related to cervical disk hernia, we also explain to patients that surgery is another possible option.

We believe that the population was large enough to show an accurate trend with reasonable statistical power. Howver, one limitation of this cross-sectional study was the lack of a demographically- matched control and /or placebo (sham irradiation) group. Another limitation is the lack of a long-term follow-up after the final treatment session to assess the latency of the efficacy. To validate the results of this study, such con-

\section{References}

1: Hattori S, Oyama M( 1974). Diagnosis of Cervical Spondylosis. A Year Book of Orthopaedic Surgery: 31-61, Kanehara Publ, Tokyo (in Japanese).

2: Eivazi B, Wiegand S, Teymoortash A,et al (2010). Laser treatment of mucosal venous malformations of the upper aerodigestive tract in 50 patients. Laser Med Sci, 25: 571-576.

3: Guzman J (2009). Neck pain and low level laser: does it work and how ? Lancet, 374: 1875-1876.

4: Quante M, Hauck M, Gromoll E, et al (2007). Dermatomal laser evoked potentials. a diagnostic approach to the dorsal root. Norm data in healthy volunteers and changes in patients with radiculopathy. Eur Spine J, 16: 943-962.

5: Quante M, Lorenz J, Hauck M (2010). Laser evoked potentials: prognostic relevance of pain pathway defects in patients with acute radiculopathy. Eur Spine J, 19: 270-278.

6: Zeredo JL, Sasaki KM, Yozgatian JH, et al (2005). Comparison of jaw opening reflexes evoked by Er:YAG laser versus scalpel incisions in Rats. Oral Surg Med Oral Radiol Endod, 100: 31-35.

7: Konstantinovic LM, Cutovic MR, Milivanvic AN, et al (2010). Low level laser therapy for acute neck pain with radiculopathy. Pain Med, 11: 1169-1178.

8: Peres MF, Luccetti G (2010). Clinical trials reportLow level laser therapy in the management of of neck pain. Curr Pain Headache Rep, 14: 325-327.

9: Caffier PP, Frieler K, Scherer B, et al (2008). Rhinitis medicamentosa: therapeutic effect of diode laser inferior turbinate reduction on nasal obstruction and decongestant abuse. Am J Rhinol 22: 433443. trolled and placebo studies with a long-term follow-up are required and warranted in the future.

\section{Conclusion}

Our results in the present study confirmed that LLLT was an effective treatment for pain related to cervical disk hernia. Moreover, for patients to continue to reap the benefits of LLLT treatment, we discovered that the education on the patients' posture during their ADL was also very important.

10: Schuller BK, Neugebauer EA (2008). Evidence for laser acupuncture in cases of orthopedic diseases: a systematic review. Schmerz, 22: 9-15.

11: Rebeiz E, April MM, Bohigian RK, et al (1991). NdYAG laser treatment of venous malformation of the head and neck. Otolaryngol Head Neck Surg 105: 655-661.

12: Synder-Mackler L, Barry AJ, Perkins AL,e t al(1989). Effects of helium- neon laser irradiation on skin resistance and pain in patients with trigger points in the neck or back. Phys Ther 69: 336-341.

13: Akai M (2006). Effect of LLLT from a viewpoint of EBM. Japan Laser Therapy Association 5:20-24 (in Japanese).

14: Arun Maiya G, Sagar MS, Fermandes D 2006). Effect of low level helium- neon laser therapy in the prevention and treatment of radiation induced mucositis in head and neck cancer patients. Indian J Med Res 124: 399- 402.

15: Shiri R, Viikali Juntura E( 2010). Low level laser therapy for neck pain. Lancet 375: 721-722.

16: Dundar U, Evcik D, Samli F, et al (2007). The effect of gallium arsenide aluminum laser therapy in the management of cervical myofascial pain syndrome: a double-blind placebo-controlled study. Clin Rheumatol, 2007; 26(6): 930-934.

17: Leaver AM, Refshauge KM, Maher CG, et al ( 2010). Conservative interventions provide shortterm relief for non specific neck pain. Physiother 56: 73-85.

18: Hurwitz EL, Carragee EJ, van der Veide G, et al (2009). Treatment of neck pain: noninvasive interventions. Manipulative Physiol Ther 32: 141-157. 